\title{
Computation of growth rates of composite entire and meromorphic functions from the view point of their relative $L^{*}$-orders
}

\author{
Sanjib Kumar Datta ${ }^{1}$, Tanmay Biswas ${ }^{2}$, Pulak Sahoo ${ }^{3}$ \\ ${ }^{1}$ Department of Mathematics, University of Kalyani, P.O.-Kalyani, Dist.-Nadia, PIN- 741235, West Bengal, India. \\ ${ }^{2}$ Rajbari, Rabindrapalli, R. N. Tagore Road, P.O.-Krishnagar, Dist.-Nadia, PIN-741101, West Bengal, India. \\ ${ }^{3}$ Department of Mathematics, University of Kalyani, P.O.-Kalyani, Dist.-Nadia, PIN- 741235, West Bengal, India.
}

Received: 18 June 2016, Accepted: 14 august 2016

Published online: 5 January 2017.

\begin{abstract}
In the paper we establish some new results depending on the comparative growth properties of composite entire or meromorphic functions using relative $L^{*}$-order and relative $L^{*}$-lower order as compared to their corresponding left and right factors.

Keywords: Entire function, meromorphic function, composition, growth, relative $L^{*}$-order, relative $L^{*}$-lower order, slowly changing function.
\end{abstract}

\section{Introduction, Definitions and Notations.}

We denote by $\mathbb{C}$ the set of all finite complex numbers. Let $f$ be a meromorphic function defined on $\mathbb{C}$. We use the standard notations and definitions in the theory of entire and meromorphic functions which are available in [2] and [7]. In

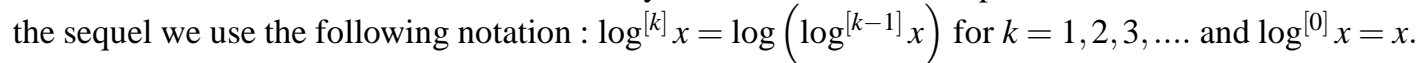

The following definition is well known.

Definition 1. The order $\rho_{f}$ and lower order $\lambda_{f}$ of an entire function $f$ are defined as

$$
\rho_{f}=\limsup _{r \rightarrow \infty} \frac{\log ^{[2]} M_{f}(r)}{\log r} \text { and } \lambda_{f}=\liminf _{r \rightarrow \infty} \frac{\log ^{[2]} M_{f}(r)}{\log r} \text {, }
$$

when $f$ is meromorphic, one can easily verify that

$$
\rho_{f}=\limsup _{r \rightarrow \infty} \frac{\log T_{f}(r)}{\log r} \text { and } \lambda_{f}=\liminf _{r \rightarrow \infty} \frac{\log T_{f}(r)}{\log r} .
$$

Let $L \equiv L(r)$ be a positive continuous function increasing slowly i.e., $L(a r) \sim L(r)$ as $r \rightarrow \infty$ for every positive constant a. Singh and Barker [5] defined it in the following way.

Definition 2. [5] A positive continuous function $L(r)$ is called a slowly changing function if for $\varepsilon(>0)$,

$$
\frac{1}{k^{\varepsilon}} \leq \frac{L(k r)}{L(r)} \leq k^{\varepsilon} \text { for } r \geq r(\varepsilon) \text { and }
$$


uniformly for $k(\geq 1)$. If further, $L(r)$ is differentiable, the above condition is equivalent to

$$
\lim _{r \rightarrow \infty} \frac{r L^{\prime}(r)}{L(r)}=0
$$

Somasundaram and Thamizharasi [6] introduced the notions of $L$-order and $L$-lower order for entire functions. The more generalised concept for $L$-order and $L$-lower order for entire and meromorphic functions are $L^{*}$-order and $L^{*}-$ lower order respectively. Their definitions are as follows:

Definition 3. [6] The $L^{*}$-order $\rho_{f}^{L^{*}}$ and the $L^{*}$-lower order $\lambda_{f}^{L^{*}}$ of an entire function $f$ are defined as

$$
\rho_{f}^{L^{*}}=\limsup _{r \rightarrow \infty} \frac{\log ^{[2]} M_{f}(r)}{\log \left[r e^{L(r)}\right]} \text { and } \lambda_{f}^{L^{*}}=\liminf _{r \rightarrow \infty} \frac{\log ^{[2]} M_{f}(r)}{\log \left[r e^{L(r)}\right]},
$$

when $f$ is meromorphic, one can easily verify that

$$
\rho_{f}^{L^{*}}=\limsup _{r \rightarrow \infty} \frac{\log T_{f}(r)}{\log \left[r e^{L(r)}\right]} \text { and } \lambda_{f}^{L^{*}}=\liminf _{r \rightarrow \infty} \frac{\log T_{f}(r)}{\log \left[r e^{L(r)}\right]} .
$$

For an entire function $g$, the Nevanlinna's characteristic function $T_{g}(r)=\frac{1}{2 \pi} \int_{0}^{2 \pi} \log ^{+}\left|g\left(r e^{i \theta}\right)\right| d \theta$ where $\log ^{+} x=\max (0, \log x)$ for $x>0$.If $g$ is non-constant then $T_{g}(r)$ is strictly increasing and continuous and its inverse $T_{g}^{-1}:\left(T_{g}(0), \infty\right) \rightarrow(0, \infty)$ exists and is such that $\lim _{s \rightarrow \infty} T_{g}^{-1}(s)=\infty$.

Lahiri and Banerjee [4] introduced the definition of relative order of a meromorphic function with respect to an entire function which is as follows.

Definition 4. [4] Let $f$ be meromorphic and $g$ be entire. The relative order of $f$ with respect to $g$ denoted by $\rho_{g}(f)$ is defined as

$$
\begin{aligned}
\rho_{g}(f) & =\inf \left\{\mu>0: T_{f}(r)<T_{g}\left(r^{\mu}\right) \text { for all sufficiently large } r\right\} \\
& =\limsup _{r \rightarrow \infty} \frac{\log T_{g}^{-1} T_{f}(r)}{\log r} .
\end{aligned}
$$

The definition coincides with the classical one [4] if $g(z)=\exp z$.

Similarly one can define the relative lower order of a meromorphic function $f$ with respect to an entire $g$ denoted by $\lambda_{g}(f)$ in the following manner :

$$
\lambda_{g}(f)=\liminf _{r \rightarrow \infty} \frac{\log T_{g}^{-1} T_{f}(r)}{\log r} .
$$

In the line of Somasundaram and Thamizharasi [6] and Lahiri and Banerjee [4] one may define the relative $L^{*}$-order and relative $L^{*}$-lower order of a meromorphic function $f$ with respect to an entire function $g$ in the following manner.

Definition 5. The relative $L^{*}$-order $\rho_{g}^{L^{*}}(f)$ and the relative $L^{*}$-lower order $\lambda_{g}^{L^{*}}(f)$ of a meromorphic function $f$ with respect to an entire function $g$ are defined by

$$
\rho_{g}^{L^{*}}(f)=\limsup _{r \rightarrow \infty} \frac{\log T_{g}^{-1} T_{f}(r)}{\log \left[r e^{L(r)}\right]} \text { and } \lambda_{g}^{L^{*}}(f)=\liminf _{r \rightarrow \infty} \frac{\log T_{g}^{-1} T_{f}(r)}{\log \left[r e^{L(r)}\right]} .
$$

In this paper we study some growth properties of composition of entire and meromorphic functions with respect to relative $L^{*}$-order and relative $L^{*}$-lower order as compared to the corresponding left and right factors. 


\section{Lemmas}

In this section we present a lemma which will be needed in the sequel.

Lemma 1. [1] Let $f$ be meromorphic and $g$ be entire and suppose that $0<\mu<\rho_{g} \leq \infty$. Then for a sequence of values of $r$ tending to infinity,

$$
T_{f \circ g}(r) \geq T_{f}\left(\exp (r)^{\mu}\right)
$$

\section{Theorems}

In this section we present the main results of the paper.

Theorem 1. Let $f$ be a meromorphic function and $g$, h be entire such that $0<\lambda_{h}^{L^{*}}(f) \leq \rho_{h}^{L^{*}}(f)<\infty$. Then for any A $>0$,

$$
\limsup _{r \rightarrow \infty} \frac{\log ^{[2]} T_{h}^{-1} T_{f \circ g}\left(\exp \left(r^{A}\right)\right)}{\log T_{h}^{-1} T_{f}\left(\exp \left(r^{\mu}\right)\right)+K(r, A ; L)}=\infty,
$$

where $0<\mu<\rho_{g}$ and

$$
K(r, A ; L)=\left\{\begin{array}{r}
0 \text { if } r^{\mu}=o\left\{L\left(\exp \left(\exp \left(\mu r^{A}\right)\right)\right)\right\} \\
\text { as } r \rightarrow \infty \\
L\left(\exp \left(\exp \left(\mu r^{A}\right)\right)\right) \text { otherwise }
\end{array}\right.
$$

Proof. Let $0<\mu<\mu^{\prime}<\rho_{g}$. Since $T_{h}^{-1}$ is an increasing functions, from the definition of relative $L^{*}$-lower order we obtain in view of Lemma 1 , for a sequence of values of $r$ tending to infinity that

$$
\begin{aligned}
\log T_{h}^{-1} T_{f \circ g}\left(\exp \left(r^{A}\right)\right) & \geq \log T_{h}^{-1} T_{f}\left(\exp \left(\exp \left(r^{A}\right)\right)^{\mu^{\prime}}\right) \\
& \geq\left(\lambda_{h}^{L^{*}}(f)-\varepsilon\right) \cdot \log \left\{\exp \left(\exp \left(r^{A}\right)\right)^{\mu^{\prime}} \cdot \exp L\left(\exp \left(\exp \left(r^{A}\right)\right)^{\mu^{\prime}}\right)\right\} \\
& \geq\left(\lambda_{h}^{L^{*}}(f)-\varepsilon\right) \cdot\left\{\left(\exp \left(r^{A}\right)\right)^{\mu^{\prime}}+L\left(\exp \left(\exp \left(r^{A}\right)\right)^{\mu^{\prime}}\right)\right\} \\
& \geq\left(\lambda_{h}^{L^{*}}(f)-\varepsilon\right) \cdot\left\{\left(\exp \left(r^{A}\right)\right)^{\mu^{\prime}}\left(1+\frac{L\left(\exp \left(\exp \left(r^{A}\right)\right)^{\mu^{\prime}}\right)}{\left(\exp \left(r^{A}\right)\right)^{\mu^{\prime}}}\right)\right\} \\
\log ^{[2]} T_{h}^{-1} T_{f \circ g}\left(\exp \left(r^{A}\right)\right) & \geq O(1)+\mu^{\prime} \log \exp \left(r^{A}\right)+\log \left\{1+\frac{L\left(\exp \left(\exp \left(r^{A}\right)\right)^{\mu^{\prime}}\right)}{\left.\left(\exp \left(r^{A}\right)\right)^{\mu^{\prime}}\right\}}\right. \\
& \geq O(1)+\mu^{\prime} r^{A}+\log \left\{1+\frac{L\left(\exp \left(\exp \left(r^{A}\right)\right)^{\mu^{\prime}}\right)}{\left.\left(\exp \left(r^{A}\right)\right)^{\mu^{\prime}}\right\}}\right. \\
& \geq O(1)+\mu^{\prime} r^{A}+\log \left[1+\frac{L\left(\exp \left(\exp \left(\mu^{\prime} r^{A}\right)\right)\right)}{\exp \left(\mu^{\prime} r^{A}\right)}\right] \\
\geq & O(1)+\mu^{\prime} r^{A}+L\left(\exp \left(\exp \left(\mu r^{A}\right)\right)\right)-\log \left[\exp \left\{L\left(\exp \left(\exp \left(\mu r^{A}\right)\right)\right)\right\}\right] \\
& +\log \left[1+\frac{L\left(\exp \left(\exp \left(\mu^{\prime} r^{A}\right)\right)\right)}{\exp \left(\mu^{\prime} r^{A}\right)}\right]
\end{aligned}
$$




$$
\begin{aligned}
\log ^{[2]} T_{h}^{-1} T_{f \circ g}\left(\exp \left(r^{A}\right)\right) \geq & O(1)+\mu^{\prime} r^{A}+L\left(\exp \left(\exp \left(\mu r^{A}\right)\right)\right)+\log \left[\frac{1}{\exp \left\{L\left(\exp \left(\exp \left(\mu r^{A}\right)\right)\right)\right\}}\right. \\
& \left.+\frac{L\left(\exp \left(\exp \left(\mu^{\prime} r^{A}\right)\right)\right)}{\exp \left\{L\left(\exp \left(\exp \left(\mu r^{A}\right)\right)\right)\right\} \cdot \exp \left(\mu^{\prime} r^{A}\right)}\right] \\
& \geq O(1)+\mu^{\prime} r^{(A-\mu)} \cdot r^{\mu}+L\left(\exp \left(\exp \left(\mu r^{A}\right)\right)\right) .
\end{aligned}
$$

Again we have for all sufficiently large values of $r$ that

$$
\begin{aligned}
\log T_{h}^{-1} T_{f}\left(\exp \left(r^{\mu}\right)\right) & \leq\left(\rho_{h}^{L^{*}}(f)+\varepsilon\right) \log \left\{\exp \left(r^{\mu}\right) e^{L\left(\exp \left(r^{\mu}\right)\right)}\right\} \\
& \leq\left(\rho_{h}^{L^{*}}(f)+\varepsilon\right)\left\{\log \exp \left(r^{\mu}\right)+L\left(\exp \left(r^{\mu}\right)\right)\right\} \\
& \leq\left(\rho_{h}^{L^{*}}(f)+\varepsilon\right)\left\{r^{\mu}+L\left(\exp \left(r^{\mu}\right)\right)\right\} .
\end{aligned}
$$

Thus,

$$
\frac{\log T_{h}^{-1} T_{f}\left(\exp \left(r^{\mu}\right)\right)-\left(\rho_{h}^{L^{*}}(f)+\varepsilon\right) L\left(\exp \left(r^{\mu}\right)\right)}{\left(\rho_{h}^{L^{*}}(f)+\varepsilon\right)} \leq r^{\mu} .
$$

Now from (1) and (2) it follows for a sequence of values of $r$ tending to infinity that

$$
\begin{aligned}
\log ^{[2]} T_{h}^{-1} T_{f \circ g}\left(\exp \left(r^{A}\right)\right) & \geq O(1)+\left(\frac{\mu^{\prime} r^{(A-\mu)}}{\rho_{h}^{L^{*}}(f)+\varepsilon}\right)\left[\log T_{h}^{-1} T_{f}\left(\exp \left(r^{\mu}\right)\right)-\left(\rho_{h}^{L^{*}}(f)+\varepsilon\right) L\left(\exp \left(r^{\mu}\right)\right)\right] \\
& +L\left(\exp \left(\exp \left(\mu r^{A}\right)\right)\right) .
\end{aligned}
$$

Therefore

$$
\frac{\log ^{[2]} T_{h}^{-1} T_{f \circ g}\left(\exp \left(r^{A}\right)\right)}{\log T_{h}^{-1} T_{f}\left(\exp \left(r^{\mu}\right)\right)} \geq \frac{L\left(\exp \left(\exp \left(\mu r^{A}\right)\right)\right)+O(1)}{\log T_{h}^{-1} T_{f}\left(\exp \left(r^{\mu}\right)\right)}+\frac{\mu^{\prime} r^{(A-\mu)}}{\rho_{h}^{L^{*}}(f)+\varepsilon}\left\{1-\frac{\left(\rho_{h}^{L^{*}}(f)+\varepsilon\right) L\left(\exp \left(r^{\mu}\right)\right)}{\log T_{h}^{-1} T_{f}\left(\exp \left(r^{\mu}\right)\right)}\right\} .
$$

Again from (3) we get for a sequence of values of $r$ tending to infinity that

$$
\begin{aligned}
\frac{\log { }^{[2]} T_{h}^{-1} T_{f \circ g}\left(\exp \left(r^{A}\right)\right)}{\log T_{h}^{-1} T_{f}\left(\exp \left(r^{\mu}\right)\right)+L\left(\exp \left(\exp \left(\mu r^{A}\right)\right)\right)} & \geq \frac{O(1)-\mu^{\prime} r^{(A-\mu)} L\left(\exp \left(r^{\mu}\right)\right)}{\log T_{h}^{-1} T_{f}\left(\exp \left(r^{\mu}\right)\right)+L\left(\exp \left(\exp \left(\mu r^{A}\right)\right)\right)} \\
& +\frac{\left(\frac{\mu^{\prime} r^{(A-\mu)}}{\rho_{h}^{L^{*}}(f)+\varepsilon}\right) \log T_{h}^{-1} T_{f}\left(\exp \left(r^{\mu}\right)\right)}{\log T_{h}^{-1} T_{f}\left(\exp \left(r^{\mu}\right)\right)+L\left(\exp \left(\exp \left(\mu r^{A}\right)\right)\right)} \\
& +\frac{L\left(\exp \left(\exp \left(\mu r^{A}\right)\right)\right)}{\log T_{h}^{-1} T_{f}\left(\exp \left(r^{\mu}\right)\right)+L\left(\exp \left(\exp \left(\mu r^{A}\right)\right)\right)}
\end{aligned}
$$

Therefore

$$
\begin{gathered}
\frac{\log ^{[2]} T_{h}^{-1} T_{f \circ g}\left(\exp \left(r^{A}\right)\right)}{\log T_{h}^{-1} T_{f}\left(\exp \left(r^{\mu}\right)\right)+L\left(\exp \left(\exp \left(\mu r^{A}\right)\right)\right)} \geq \frac{\frac{O(1)-\mu^{\prime} r^{(A-\mu)} L\left(\exp \left(r^{\mu}\right)\right)}{L\left(\exp \left(\exp \left(\mu r^{A}\right)\right)\right)}}{\frac{\log T_{h}^{-1} T_{f}\left(\exp \left(r^{\mu}\right)\right)}{L\left(\exp \left(\exp \left(\mu r^{A}\right)\right)\right)}+1} \\
+\frac{\left(\frac{\mu^{\prime} r^{(A-\mu)}}{\rho_{h}^{*}(f)+\varepsilon}\right) \log T_{h}^{-1} T_{f}\left(\exp \left(r^{\mu}\right)\right)}{1+\frac{L\left(\exp \left(\exp \left(\mu r^{A}\right)\right)\right)}{\log T_{h}^{-1} T_{f}\left(\exp \left(r^{\mu}\right)\right)}}+\frac{1}{1+\frac{\log T_{h}^{-1} T_{f}\left(\exp \left(r^{\mu}\right)\right)}{L\left(\exp \left(\exp \left(\mu r^{A}\right)\right)\right)}}
\end{gathered}
$$


Case 1. If $r^{\mu}=o\left\{L\left(\exp \left(\exp \left(\mu r^{A}\right)\right)\right)\right\}$ then it follows from (4) that

$$
\underset{r \rightarrow \infty}{\limsup } \frac{\log ^{[2]} T_{h}^{-1} T_{f \circ g}\left(\exp \left(r^{A}\right)\right)}{\log T_{h}^{-1} T_{f}\left(\exp \left(r^{\mu}\right)\right)}=\infty .
$$

Case 2. $r^{\mu} \neq o\left\{L\left(\exp \left(\exp \left(\mu r^{A}\right)\right)\right)\right\}$ then two sub cases may arise.

Case 2.1 If $L\left(\exp \left(\exp \left(\mu r^{A}\right)\right)\right)=o\left\{\log T_{h}^{-1} T_{f}\left(\exp \left(r^{\mu}\right)\right)\right\}$, then we get from (5) that

$$
\limsup _{r \rightarrow \infty} \frac{\log ^{[2]} T_{h}^{-1} T_{f \circ g}\left(\exp \left(r^{A}\right)\right)}{\log T_{h}^{-1} T_{f}\left(\exp \left(r^{\mu}\right)\right)+L\left(\exp \left(\exp \left(\mu r^{A}\right)\right)\right)}=\infty .
$$

Case 2.2 If $L\left(\exp \left(\exp \left(\mu r^{\rho_{g}^{*}}\right)\right)\right) \sim \log T_{h}^{-1} T_{f}\left(\exp \left(r^{\mu}\right)\right)$ then

$$
\lim _{r \rightarrow \infty} \frac{L\left\{\exp \left(\exp \left(\mu r^{A}\right)\right)\right\}}{\log T_{h}^{-1} T_{f}\left(\exp \left(r^{\mu}\right)\right)}=1
$$

and we obtain from (5) that

$$
\limsup _{r \rightarrow \infty} \frac{\log ^{[2]} T_{h}^{-1} T_{f \circ g}\left(\exp \left(r^{A}\right)\right)}{\log T_{h}^{-1} T_{f}\left(\exp \left(r^{\mu}\right)\right)+L\left(\exp \left(\exp \left(\mu r^{A}\right)\right)\right)}=\infty .
$$

Combining Case I and Case II we may obtain that

$$
\limsup _{r \rightarrow \infty} \frac{\log ^{[2]} T_{h}^{-1} T_{f \circ g}\left(\exp \left(r^{A}\right)\right)}{\log T_{h}^{-1} T_{f}\left(\exp \left(r^{\mu}\right)\right)+L\left(\exp \left(\exp \left(\mu r^{A}\right)\right)\right)}=\infty,
$$

where

$$
K(r, A ; L)=\left\{\begin{array}{r}
0 \text { if } r^{\mu}=o\left\{L\left(\exp \left(\exp \left(\mu r^{A}\right)\right)\right)\right\} \\
\text { as } r \rightarrow \infty \\
L\left(\exp \left(\exp \left(\mu r^{A}\right)\right)\right) \text { otherwise }
\end{array}\right.
$$

This proves the theorem.

Theorem 2. Let $f$ be a meromorphic function and $g, h$ be entire such that $\lambda_{h}^{L^{*}}(f)>0$ and $\rho_{h}^{L^{*}}(g)<\infty$. Then for any $A>0$

$$
\limsup _{r \rightarrow \infty} \frac{\log ^{[2]} T_{h}^{-1} T_{f \circ g}\left(\exp \left(r^{A}\right)\right)}{\log T_{h}^{-1} T_{g}\left(\exp \left(r^{\mu}\right)\right)+K(r, A ; L)}=\infty,
$$

where $0<\mu<\rho_{g}$ and

$$
K(r, A ; L)=\left\{\begin{array}{r}
0 \text { if } r^{\mu}=o\left\{L\left(\exp \left(\exp \left(\mu r^{A}\right)\right)\right)\right\} \\
\text { as } r \rightarrow \infty \\
L\left(\exp \left(\exp \left(\mu r^{A}\right)\right)\right) \text { otherwise }
\end{array}\right.
$$

The proof is omitted because it can be carried out in the line of Theorem 1.

Theorem 3. Let $f$ be meromorphic and $g$ and $h$ be any two entire functions such that $0<\lambda_{h}^{L^{*}}(f \circ g) \leq \rho_{h}^{L^{*}}(f \circ g)<\infty$ and $0<\lambda_{h}^{L^{*}}(f) \leq \rho_{h}^{L^{*}}(f)<\infty$. If $L\left(r^{A}\right)=o\left\{\log T_{h}^{-1} T_{f}\left(r^{A}\right)\right\}$ as $r \rightarrow \infty$ then for any positive number $A$,

$$
\frac{\lambda_{h}^{L^{*}}(f \circ g)}{A \rho_{h}^{L^{*}}(f)} \leq \liminf _{r \rightarrow \infty} \frac{\log T_{h}^{-1} T_{f \circ g}(r)}{\log T_{h}^{-1} T_{f}\left(r^{A}\right)+L\left(r^{A}\right)} \leq \frac{\lambda_{h}^{L^{*}}(f \circ g)}{A \lambda_{h}^{L^{*}}(f)} \leq \limsup _{r \rightarrow \infty} \frac{\log T_{h}^{-1} T_{f \circ g}(r)}{\log T_{h}^{-1} T_{f}\left(r^{A}\right)+L\left(r^{A}\right)} \leq \frac{\rho_{h}^{L^{*}}(f \circ g)}{A \lambda_{h}^{L^{*}}(f)} .
$$


Proof. From the definition of relative $L^{*}$-order and relative $L^{*}$-lower order of a meromorphic function with respect to an entire function we have for arbitrary positive $\varepsilon$ and for all sufficiently large values of $r$ that

$$
\begin{aligned}
\log T_{h}^{-1} T_{f \circ g}(r) & \geq\left(\lambda_{h}^{L^{*}}(f \circ g)-\varepsilon\right) \log \left\{r e^{L(r)}\right\} \\
& \geq\left(\lambda_{h}^{L^{*}}(f \circ g)-\varepsilon\right)\{\log r+L(r)\} \\
& \geq\left(\lambda_{h}^{L^{*}}(f \circ g)-\varepsilon\right)\left\{\log r+\frac{1}{A} L\left(r^{A}\right)\right\}+\left(\lambda_{h}^{L^{*}}(f \circ g)-\varepsilon\right)\left\{L(r)-\frac{1}{A} L\left(r^{A}\right)\right\}
\end{aligned}
$$

and

$$
\begin{aligned}
\log T_{h}^{-1} T_{f}\left(r^{A}\right) & \leq\left(\rho_{h}^{L^{*}}(f)+\varepsilon\right) \log \left\{r^{A} e^{L\left(r^{A}\right)}\right\} \\
& \leq\left(\rho_{h}^{L^{*}}(f)+\varepsilon\right)\left\{A \log r+L\left(r^{A}\right)\right\}
\end{aligned}
$$

So we have,

$$
\frac{\log T_{h}^{-1} T_{f}\left(r^{A}\right)}{A\left(\rho_{h}^{L^{*}}(f)+\varepsilon\right)} \leq \log r+\frac{1}{A} L\left(r^{A}\right)
$$

Now from (6) and (7) it follows for all sufficiently large values of $r$ that

$$
\log T_{h}^{-1} T_{f \circ g}(r) \geq \frac{\left(\lambda_{h}^{L^{*}}(f \circ g)-\varepsilon\right)}{A\left(\rho_{h}^{L^{*}}(f)+\varepsilon\right)} \log T_{h}^{-1} T_{f}\left(r^{A}\right)+\left(\lambda_{h}^{L^{*}}(f \circ g)-\varepsilon\right)\left\{L(r)-\frac{1}{A} L\left(r^{A}\right)\right\},
$$

Therefore we write

$$
\frac{\log T_{h}^{-1} T_{f \circ g}(r)}{\log T_{h}^{-1} T_{f}\left(r^{A}\right)+L\left(r^{A}\right)} \geq \frac{\left(\lambda_{h}^{L^{*}}(f \circ g)-\varepsilon\right)}{A\left(\rho_{h}^{L^{*}}(f)+\varepsilon\right)} \cdot \frac{\log T_{h}^{-1} T_{f}\left(r^{A}\right)}{\log T_{h}^{-1} T_{f}\left(r^{A}\right)+L\left(r^{A}\right)}+\frac{\left(\lambda_{h}^{L^{*}}(f \circ g)-\varepsilon\right)\left\{L(r)-\frac{1}{A} L\left(r^{A}\right)\right\}}{\log T_{h}^{-1} T_{f}\left(r^{A}\right)+L\left(r^{A}\right)}
$$

Finally

$$
\frac{\log T_{h}^{-1} T_{f \circ g}(r)}{\log T_{h}^{-1} T_{f}\left(r^{A}\right)+L\left(r^{A}\right)} \geq \frac{\frac{\lambda_{h}^{L^{*}}(f \circ g)-\varepsilon}{A\left(\rho_{h}^{L^{*}}(f)+\varepsilon\right)}}{1+\frac{L\left(r^{A}\right)}{\log T_{h}^{-1} T_{f}\left(r^{A}\right)}}+\frac{\left(\lambda_{h}^{L^{*}}(f \circ g)-\varepsilon\right)\left\{\frac{L(r)}{L\left(r^{A}\right)}-\frac{1}{A}\right\}}{1+\frac{\log T_{h}^{-1} T_{f}\left(r^{A}\right)}{L\left(r^{A}\right)}} .
$$

Since $L\left(r^{A}\right)=o\left\{\log T_{h}^{-1} T_{f}\left(r^{A}\right)\right\}$ as $r \rightarrow \infty$, it follows from (8) that

$$
\liminf _{r \rightarrow \infty} \frac{\log T_{h}^{-1} T_{f \circ g}(r)}{\log T_{h}^{-1} T_{f}\left(r^{A}\right)+L\left(r^{A}\right)} \geq \frac{\left(\lambda_{h}^{L^{*}}(f \circ g)-\varepsilon\right)}{A\left(\rho_{h}^{L^{*}}(f)+\varepsilon\right)} .
$$

As $\varepsilon(>0)$ is arbitrary, we get from (9) that

$$
\liminf _{r \rightarrow \infty} \frac{\log T_{h}^{-1} T_{f \circ g}(r)}{\log T_{h}^{-1} T_{f}\left(r^{A}\right)+L\left(r^{A}\right)} \geq \frac{\lambda_{h}^{L^{*}}(f \circ g)}{A \rho_{h}^{L^{*}}(f)} .
$$


Again for a sequence of values of $r$ tending to infinity,

$$
\begin{aligned}
\log T_{h}^{-1} T_{f \circ g}(r) & \leq\left(\lambda_{h}^{L^{*}}(f \circ g)+\varepsilon\right) \log \left\{r e^{L(r)}\right\} \\
& \leq\left(\lambda_{h}^{L^{*}}(f \circ g)+\varepsilon\right)\left\{\log r+\frac{1}{A} L\left(r^{A}\right)\right\}+\left(\lambda_{h}^{L^{*}}(f \circ g)+\varepsilon\right)\left\{L(r)-\frac{1}{A} L\left(r^{A}\right)\right\}
\end{aligned}
$$

and for all sufficiently large values of $r$,

$$
\begin{aligned}
\log T_{h}^{-1} T_{f}\left(r^{A}\right) & \geq\left(\lambda_{h}^{L^{*}}(f)-\varepsilon\right) \log \left\{r^{A} e^{L\left(r^{A}\right)}\right\} \\
& \geq\left(\lambda_{h}^{L^{*}}(f)-\varepsilon\right)\left\{A \log r+L\left(r^{A}\right)\right\} .
\end{aligned}
$$

We obtain,

$$
\frac{\log T_{h}^{-1} T_{f}\left(r^{A}\right)}{A\left(\lambda_{h}^{L^{*}}(f)-\varepsilon\right)} \geq \log r+\frac{1}{A} L\left(r^{A}\right)
$$

Combining (11) and (12) we get for a sequence of values of $r$ tending to infinity that

$$
\log T_{h}^{-1} T_{f \circ g}(r) \leq \frac{\left(\lambda_{h}^{L^{*}}(f \circ g)+\varepsilon\right)}{A\left(\lambda_{h}^{L^{*}}(f)-\varepsilon\right)} \log T_{h}^{-1} T_{f}\left(r^{A}\right)+\left(\lambda_{h}^{L^{*}}(f \circ g)+\varepsilon\right)\left\{L(r)-\frac{1}{A} L\left(r^{A}\right)\right\} .
$$

That is,

$$
\frac{\log T_{h}^{-1} T_{f \circ g}(r)}{\log T_{h}^{-1} T_{f}\left(r^{A}\right)+L\left(r^{A}\right)} \leq \frac{\lambda_{h}^{L^{*}}(f \circ g)+\varepsilon}{A\left(\lambda_{h}^{L^{*}}(f)-\varepsilon\right)} \cdot \frac{\log T_{h}^{-1} T_{f}\left(r^{A}\right)}{\log T_{h}^{-1} T_{f}\left(r^{A}\right)+L\left(r^{A}\right)}+\frac{\left(\lambda_{h}^{L^{*}}(f \circ g)+\varepsilon\right)\left\{L(r)-\frac{1}{A} L\left(r^{A}\right)\right\}}{\log T_{h}^{-1} T_{f}\left(r^{A}\right)+L\left(r^{A}\right)} .
$$

Therefore,

$$
\frac{\log T_{h}^{-1} T_{f \circ g}(r)}{\log T_{h}^{-1} T_{f}\left(r^{A}\right)+L\left(r^{A}\right)} \leq \frac{\frac{\lambda_{h}^{L^{*}}(f \circ g)+\varepsilon}{A\left(\lambda_{h}^{L^{*}}(f)-\varepsilon\right)}}{1+\frac{L\left(r^{A}\right)}{\log T_{h}^{-1} T_{f}\left(r^{A}\right)}}+\frac{\left(\lambda_{h}^{L^{*}}(f \circ g)+\varepsilon\right)\left\{\frac{L(r)}{L\left(r^{A}\right)}-\frac{1}{A}\right\}}{1+\frac{\log T_{h}^{-1} T_{f}\left(r^{A}\right)}{L\left(r^{A}\right)}} .
$$

As $L\left(r^{A}\right)=o\left\{\log T_{h}^{-1} T_{f}\left(r^{A}\right)\right\}$ as $r \rightarrow \infty$ we get from (13) that

$$
\liminf _{r \rightarrow \infty} \frac{\log T_{h}^{-1} T_{f \circ g}(r)}{\log T_{h}^{-1} T_{f}\left(r^{A}\right)+L\left(r^{A}\right)} \leq \frac{\lambda_{h}^{L^{*}}(f \circ g)+\varepsilon}{A\left(\lambda_{h}^{L^{*}}(f)-\varepsilon\right)} .
$$

Since $\varepsilon(>0)$ is arbitrary, it follows from (14) that

$$
\liminf _{r \rightarrow \infty} \frac{\log T_{h}^{-1} T_{f \circ g}(r)}{\log T_{h}^{-1} T_{f}\left(r^{A}\right)+L\left(r^{A}\right)} \leq \frac{\lambda_{h}^{L^{*}}(f \circ g)}{A \lambda_{h}^{L^{*}}(f)} .
$$

Also for a sequence of values of $r$ tending to infinity,

$$
\begin{aligned}
\log T_{h}^{-1} T_{f}\left(r^{A}\right) & \leq\left(\lambda_{h}^{L^{*}}(f)+\varepsilon\right) \log \left\{r^{A} e^{L\left(r^{A}\right)}\right\} \\
& \leq\left(\lambda_{h}^{L^{*}}(f)+\varepsilon\right)\left\{A \log r+L\left(r^{A}\right)\right\},
\end{aligned}
$$


And we have,

$$
\text { i.e., } \frac{\log T_{h}^{-1} T_{f}\left(r^{A}\right)}{A\left(\lambda_{h}^{L^{*}}(f)+\varepsilon\right)} \leq \log r+\frac{1}{A} L\left(r^{A}\right)
$$

Now from (6) and (16) we obtain for a sequence of values of $r$ tending to infinity that

$$
\log T_{h}^{-1} T_{f \circ g}(r) \geq \frac{\left(\lambda_{h}^{L^{*}}(f \circ g)-\varepsilon\right)}{A\left(\lambda_{h}^{L^{*}}(f)+\varepsilon\right)} \log T_{h}^{-1} T_{f}\left(r^{A}\right)+\left(\lambda_{h}^{L^{*}}(f \circ g)-\varepsilon\right)\left\{L(r)-\frac{1}{A} L\left(r^{A}\right)\right\} .
$$

That is,

$$
\frac{\log T_{h}^{-1} T_{f \circ g}(r)}{\log T_{h}^{-1} T_{f}\left(r^{A}\right)+L\left(r^{A}\right)} \geq \frac{\lambda_{h}^{L^{*}}(f \circ g)-\varepsilon}{A\left(\lambda_{h}^{L^{*}}(f)+\varepsilon\right)} \cdot \frac{\log M_{h}^{-1} M_{f}\left(r^{A}\right)}{\log T_{h}^{-1} T_{f}\left(r^{A}\right)+L\left(r^{A}\right)}+\frac{\left(\lambda_{h}^{L^{*}}(f \circ g)-\varepsilon\right)\left\{L(r)-\frac{1}{A} L\left(r^{A}\right)\right\}}{\log T_{h}^{-1} T_{f}\left(r^{A}\right)+L\left(r^{A}\right)} .
$$

So we have

$$
\frac{\log T_{h}^{-1} T_{f \circ g}(r)}{\log T_{h}^{-1} T_{f}\left(r^{A}\right)+L\left(r^{A}\right)} \geq \frac{\frac{\lambda_{h}^{L^{*}}(f \circ g)-\varepsilon}{A\left(\lambda_{h}^{L^{*}}(f)+\varepsilon\right)}}{1+\frac{L\left(r^{A}\right)}{\log T_{h}^{-1} T_{f}\left(r^{A}\right)}}+\frac{\left(\lambda_{h}^{L^{*}}(f \circ g)-\varepsilon\right)\left\{\frac{L(r)}{L\left(r^{A}\right)}-\frac{1}{A}\right\}}{1+\frac{\log T_{h}^{-1} T_{f}\left(r^{A}\right)}{L\left(r^{A}\right)}} .
$$

In view of the condition $L\left(r^{A}\right)=o\left\{\log T_{h}^{-1} T_{f}\left(r^{A}\right)\right\}$ as $r \rightarrow \infty$ we obtain from (17) that

$$
\limsup _{r \rightarrow \infty} \frac{\log T_{h}^{-1} T_{f \circ g}(r)}{\log T_{h}^{-1} T_{f}\left(r^{A}\right)+L\left(r^{A}\right)} \geq \frac{\lambda_{h}^{L^{*}}(f \circ g)-\varepsilon}{A\left(\lambda_{h}^{L^{*}}(f)+\varepsilon\right)} .
$$

Since $\varepsilon(>0)$ is arbitrary, it follows from (18) that

$$
\limsup _{r \rightarrow \infty} \frac{\log T_{h}^{-1} T_{f \circ g}(r)}{\log T_{h}^{-1} T_{f}\left(r^{A}\right)+L\left(r^{A}\right)} \geq \frac{\lambda_{h}^{L^{*}}(f \circ g)}{A \lambda_{h}^{L^{*}}(f)}
$$

Also for all sufficiently large values of $r$,

$$
\begin{aligned}
\log T_{h}^{-1} T_{f \circ g}(r) & \leq\left(\rho_{h}^{L^{*}}(f \circ g)+\varepsilon\right) \log \left\{r e^{L(r)}\right\} \\
& \leq\left(\rho_{h}^{L^{*}}(f \circ g)+\varepsilon\right)\{\log r+L(r)\} \\
& \leq\left(\rho_{h}^{L^{*}}(f \circ g)+\varepsilon\right)\left\{\log r+\frac{1}{A} L\left(r^{A}\right)\right\}+\left(\rho_{h}^{L^{*}}(f \circ g)+\varepsilon\right)\left\{L(r)-\frac{1}{A} L\left(r^{A}\right)\right\} .
\end{aligned}
$$

So from (12) and (20) it follows for all sufficiently large values of $r$ that

$$
\log T_{h}^{-1} T_{f \circ g}(r) \leq \frac{\left(\rho_{h}^{L^{*}}(f \circ g)+\varepsilon\right)}{A\left(\lambda_{h}^{L^{*}}(f)-\varepsilon\right)} \log T_{h}^{-1} T_{f}\left(r^{A}\right)+L\left(r^{A}\right)+\left(\rho_{h}^{L^{*}}(f \circ g)+\varepsilon\right)\left\{L(r)-\frac{1}{A} L\left(r^{A}\right)\right\} .
$$

That is,

$$
\frac{\log T_{h}^{-1} T_{f \circ g}(r)}{\log T_{h}^{-1} T_{f}\left(r^{A}\right)+L\left(r^{A}\right)} \leq \frac{\rho_{h}^{L^{*}}(f \circ g)+\varepsilon}{A\left(\lambda_{h}^{L^{*}}(f)-\varepsilon\right)} \cdot \frac{\log T_{h}^{-1} T_{f}\left(r^{A}\right)}{\log T_{h}^{-1} T_{f}\left(r^{A}\right)+L\left(r^{A}\right)}+\frac{\left(\rho_{h}^{L^{*}}(f \circ g)+\varepsilon\right)\left\{L(r)-\frac{1}{A} L\left(r^{A}\right)\right\}}{\log T_{h}^{-1} T_{f}\left(r^{A}\right)+L\left(r^{A}\right)}
$$


So we have,

$$
\frac{\log T_{h}^{-1} T_{f \circ g}(r)}{\log T_{h}^{-1} T_{f}\left(r^{A}\right)+L\left(r^{A}\right)} \leq \frac{\frac{\rho_{h}^{L^{*}}(f \circ g)+\varepsilon}{A\left(\lambda_{h}^{L^{*}}(f)-\varepsilon\right)}}{1+\frac{L\left(r^{A}\right)}{\log T_{h}^{-1} T_{f}\left(r^{A}\right)}}+\frac{\left(\rho_{h}^{L^{*}}(f \circ g)+\varepsilon\right)\left\{\frac{L(r)}{L\left(r^{A}\right)}-\frac{1}{A}\right\}}{1+\frac{\log T_{h}^{-1} T_{f}\left(r^{A}\right)}{L\left(r^{A}\right)}} .
$$

Using $L\left(r^{A}\right)=o\left\{\log T_{h}^{-1} T_{f}\left(r^{A}\right)\right\}$ as $r \rightarrow \infty$ we obtain from (21) that

$$
\limsup _{r \rightarrow \infty} \frac{\log T_{h}^{-1} T_{f \circ g}(r)}{\log T_{h}^{-1} T_{f}\left(r^{A}\right)+L\left(r^{A}\right)} \leq \frac{\rho_{h}^{L^{*}}(f \circ g)+\varepsilon}{A\left(\lambda_{h}^{L^{*}}(f)-\varepsilon\right)} .
$$

As $\varepsilon(>0)$ is arbitrary, it follows from (22) that

$$
\limsup _{r \rightarrow \infty} \frac{\log T_{h}^{-1} T_{f \circ g}(r)}{\log T_{h}^{-1} T_{f}\left(r^{A}\right)+L\left(r^{A}\right)} \leq \frac{\rho_{h}^{L^{*}}(f \circ g)}{A \lambda_{h}^{L^{*}}(f)}
$$

Thus the theorem follows from $(10),(15),(19)$ and (23).

Similarly in view of Theorem 3 , we may state the following theorem without proof for the right factor $g$ of the composite function $f \circ g$.

Theorem 4. Let $f$ be a meromorphic and $g$ and $h$ be any two entire functions such that $0<\lambda_{h}^{L^{*}}(f \circ g) \leq \rho_{h}^{L^{*}}(f \circ g)<\infty$ and $0<\lambda_{h}^{L^{*}}(g) \leq \rho_{h}^{L^{*}}(g)<\infty$. If $L\left(r^{A}\right)=o\left\{\log T_{h}^{-1} T_{g}\left(r^{A}\right)\right\}$ as $r \rightarrow \infty$ then for any positive number $A$,

$$
\frac{\lambda_{h}^{L^{*}}(f \circ g)}{A \rho_{h}^{L^{*}}(g)} \leq \liminf _{r \rightarrow \infty} \frac{\log T_{h}^{-1} T_{f \circ g}(r)}{\log T_{h}^{-1} T_{g}\left(r^{A}\right)+L\left(r^{A}\right)} \leq \frac{\lambda_{h}^{L^{*}}(f \circ g)}{A \lambda_{h}^{L^{*}}(g)} \leq \limsup _{r \rightarrow \infty} \frac{\log T_{h}^{-1} T_{f \circ g}(r)}{\log T_{h}^{-1} T_{g}\left(r^{A}\right)+L\left(r^{A}\right)} \leq \frac{\rho_{h}^{L^{*}}(f \circ g)}{A \lambda_{h}^{L^{*}}(g)} .
$$

Theorem 5. Let $f$ be a meromorphic and $g$ and $h$ be any two entire functions with $0<\rho_{h}^{L^{*}}(f \circ g)<\infty$ and $0<\rho_{h}^{L^{*}}(f)<\infty$. If $L\left(r^{A}\right)=o\left\{\log T_{h}^{-1} T_{f}\left(r^{A}\right)\right\}$ as $r \rightarrow \infty$ then for any positive number $A$,

$$
\liminf _{r \rightarrow \infty} \frac{\log T_{h}^{-1} T_{f \circ g}(r)}{\log T_{h}^{-1} T_{f}\left(r^{A}\right)+L\left(r^{A}\right)} \leq \frac{\rho_{h}^{L^{*}}(f \circ g)}{A \rho_{h}^{L^{*}}(f)} \leq \limsup _{r \rightarrow \infty} \frac{\log T_{h}^{-1} T_{f \circ g}(r)}{\log T_{h}^{-1} T_{f}\left(r^{A}\right)+L\left(r^{A}\right)} .
$$

Proof. From the definition of $\rho_{h}^{L^{*}}(f)$, we get for a sequence of values of $r$ tending to infinity that

$$
\begin{aligned}
\log T_{h}^{-1} T_{f}\left(r^{A}\right) & \geq\left(\rho_{h}^{L^{*}}(f)-\varepsilon\right) \log \left\{r^{A} e^{L\left(r^{A}\right)}\right\} \\
& \geq\left(\rho_{h}^{L^{*}}(f)-\varepsilon\right)\left\{A \log r+L\left(r^{A}\right)\right\}
\end{aligned}
$$

That is,

$$
\frac{\log T_{h}^{-1} T_{f}\left(r^{A}\right)}{A\left(\rho_{h}^{L^{*}}(f)-\varepsilon\right)} \geq \log r+\frac{1}{A} L\left(r^{A}\right)
$$

Now from (20) and (24) it follows for a sequence of values of $r$ tending to infinity that

$$
\log T_{h}^{-1} T_{f \circ g}(r) \leq \frac{\left(\rho_{h}^{L^{*}}(f \circ g)+\varepsilon\right)}{A\left(\rho_{h}^{L^{*}}(f)-\varepsilon\right)} \log T_{h}^{-1} T_{f}\left(r^{A}\right)+\left(\rho_{h}^{L^{*}}(f \circ g)+\varepsilon\right)\left\{L(r)-\frac{1}{A} L\left(r^{A}\right)\right\} .
$$


22 BISK

S. K. Data, T. Biswas, P. Shoo: Computation of growth rates of composite entire and.......

That is,

$$
\frac{\log T_{h}^{-1} T_{f \circ g}(r)}{\log T_{h}^{-1} T_{f}\left(r^{A}\right)+L\left(r^{A}\right)} \leq \frac{\rho_{h}^{L^{*}}(f \circ g)+\varepsilon}{A\left(\rho_{h}^{L^{*}}(f)-\varepsilon\right)} \cdot \frac{\log T_{h}^{-1} T_{f}\left(r^{A}\right)}{\log T_{h}^{-1} T_{f}\left(r^{A}\right)+L\left(r^{A}\right)}+\frac{\left(\rho_{h}^{L^{*}}(f \circ g)+\varepsilon\right)\left\{L(r)-\frac{1}{A} L\left(r^{A}\right)\right\}}{\log T_{h}^{-1} T_{f}\left(r^{A}\right)+L\left(r^{A}\right)} .
$$

So we have

$$
\frac{\log T_{h}^{-1} T_{f \circ g}(r)}{\log T_{h}^{-1} T_{f}\left(r^{A}\right)+L\left(r^{A}\right)} \leq \frac{\frac{\rho_{h}^{L^{*}}(f \circ g)+\varepsilon}{A\left(\rho_{h}^{L^{*}}(f)-\varepsilon\right)}}{1+\frac{L\left(r^{A}\right)}{\log T_{h}^{-1} T_{f}\left(r^{A}\right)}}+\frac{\left(\rho_{h}^{L^{*}}(f \circ g)+\varepsilon\right)\left\{\frac{L(r)}{L\left(r^{A}\right)}-\frac{1}{A}\right\}}{1+\frac{\log T_{h}^{-1} T_{f}\left(r^{A}\right)}{L\left(r^{A}\right)}} .
$$

Using $L\left(r^{A}\right)=o\left\{\log T_{h}^{-1} T_{f}\left(r^{A}\right)\right\}$ as $r \rightarrow \infty$ we obtain from (25) that

$$
\liminf _{r \rightarrow \infty} \frac{\log T_{h}^{-1} T_{f \circ g}(r)}{\log T_{h}^{-1} T_{f}\left(r^{A}\right)+L\left(r^{A}\right)} \leq \frac{\rho_{h}^{L^{*}}(f \circ g)+\varepsilon}{A\left(\rho_{h}^{L^{*}}(f)-\varepsilon\right)} .
$$

As $\varepsilon(>0)$ is arbitrary, it follows from (26) that

$$
\liminf _{r \rightarrow \infty} \frac{\log T_{h}^{-1} T_{f \circ g}(r)}{\log T_{h}^{-1} T_{f}\left(r^{A}\right)+L\left(r^{A}\right)} \leq \frac{\rho_{h}^{L^{*}}(f \circ g)}{A \rho_{h}^{L^{*}}(f)} .
$$

Again for a sequence of values of $r$ tending to infinity,

$$
\begin{aligned}
\log T_{h}^{-1} T_{f \circ g}(r) & \geq\left(\rho_{h}^{L^{*}}(f \circ g)-\varepsilon\right) \log \left\{r e^{L(r)}\right\} \\
& \geq\left(\rho_{h}^{L^{*}}(f \circ g)-\varepsilon\right)\{\log r+L(r)\} \\
& \geq\left(\rho_{h}^{L^{*}}(f \circ g)-\varepsilon\right)\left\{\log r+\frac{1}{A} L\left(r^{A}\right)\right\}+\left(\rho_{h}^{L^{*}}(f \circ g)-\varepsilon\right)\left\{L(r)-\frac{1}{A} L\left(r^{A}\right)\right\}
\end{aligned}
$$

So combining (7) and (28) we get for a sequence of values of $r$ tending to infinity that

$$
\log T_{h}^{-1} T_{f \circ g}(r) \geq \frac{\left(\rho_{h}^{L^{*}}(f \circ g)-\varepsilon\right)}{A\left(\rho_{h}^{L^{*}}(f)+\varepsilon\right)} \log T_{h}^{-1} T_{f}\left(r^{A}\right)+\left(\rho_{h}^{L^{*}}(f \circ g)-\varepsilon\right)\left\{L(r)-\frac{1}{A} L\left(r^{A}\right)\right\},
$$

That is,

$$
\frac{\log T_{h}^{-1} T_{f \circ g}(r)}{\log T_{h}^{-1} T_{f}\left(r^{A}\right)+L\left(r^{A}\right)} \geq \frac{\left(\rho_{h}^{L^{*}}(f \circ g)-\varepsilon\right)}{A\left(\rho_{h}^{L^{*}}(f)+\varepsilon\right)} \cdot \frac{\log T_{h}^{-1} T_{f}\left(r^{A}\right)}{\log T_{h}^{-1} T_{f}\left(r^{A}\right)+L\left(r^{A}\right)}+\frac{\left(\rho_{h}^{L^{*}}(f \circ g)-\varepsilon\right)\left\{L(r)-\frac{1}{A} L\left(r^{A}\right)\right\}}{\log T_{h}^{-1} T_{f}\left(r^{A}\right)+L\left(r^{A}\right)} .
$$

So we have,

$$
\frac{\log T_{h}^{-1} T_{f \circ g}(r)}{\log T_{h}^{-1} T_{f}\left(r^{A}\right)+L\left(r^{A}\right)} \geq \frac{\frac{\rho_{h}^{L^{*}}(f \circ g)-\varepsilon}{A\left(\rho_{h}^{L^{*}}(f)+\varepsilon\right)}}{1+\frac{L\left(r^{A}\right)}{\log T_{h}^{-1} T_{f}\left(r^{A}\right)}}+\frac{\left(\rho_{h}^{L^{*}}(f \circ g)-\varepsilon\right)\left\{\frac{L(r)}{L\left(r^{A}\right)}-\frac{1}{A}\right\}}{1+\frac{\log T_{h}^{-1} T_{f}\left(r^{A}\right)}{L\left(r^{A}\right)}} .
$$

Since $L\left(r^{A}\right)=o\left\{\log T_{h}^{-1} T_{f}\left(r^{A}\right)\right\}$ as $r \rightarrow \infty$, it follows from (29) that

$$
\limsup _{r \rightarrow \infty} \frac{\log T_{h}^{-1} T_{f \circ g}(r)}{\log T_{h}^{-1} T_{f}\left(r^{A}\right)+L\left(r^{A}\right)} \geq \frac{\rho_{h}^{L^{*}}(f \circ g)-\varepsilon}{A\left(\rho_{h}^{L^{*}}(f)+\varepsilon\right)} .
$$

(C) 2017 BISKA Bilisim Technology 
As $\varepsilon(>0)$ is arbitrary, we get from $(30)$ that

$$
\limsup _{r \rightarrow \infty} \frac{\log T_{h}^{-1} T_{f \circ g}(r)}{\log T_{h}^{-1} T_{f}\left(r^{A}\right)+L\left(r^{A}\right)} \geq \frac{\rho_{h}^{L^{*}}(f \circ g)}{A \rho_{h}^{L^{*}}(f)} .
$$

Thus the theorem follows from (27) and (31).

Theorem 6. Let $f$ be a meromorphic and $g$ and $h$ be any two entire functions such that $0<\rho_{h}^{L^{*}}(f \circ g)<\infty$ and $0<$ $\rho_{h}^{L^{*}}(g)<\infty$. If $L\left(r^{A}\right)=o\left\{\log T_{h}^{-1} T_{g}\left(r^{A}\right)\right\}$ as $r \rightarrow \infty$ then for any positive number $A$,

$$
\liminf _{r \rightarrow \infty} \frac{\log T_{h}^{-1} T_{f \circ g}(r)}{\log T_{h}^{-1} T_{g}\left(r^{A}\right)+L\left(r^{A}\right)} \leq \frac{\rho_{h}^{L^{*}}(f \circ g)}{A \rho_{h}^{L^{*}}(g)} \leq \limsup _{r \rightarrow \infty} \frac{\log T_{h}^{-1} T_{f \circ g}(r)}{\log T_{h}^{-1} T_{g}\left(r^{A}\right)+L\left(r^{A}\right)} .
$$

The proof is omitted.

The following theorem is a natural consequence of Theorem 3 and Theorem 5.

Theorem 7. Let $f$ be a meromorphic and $g$ and $h$ be any two entire functions such that $0<\lambda_{h}^{L^{*}}(f \circ g) \leq \rho_{h}^{L^{*}}(f \circ g)<\infty$ and $0<\lambda_{h}^{L^{*}}(f) \leq \rho_{h}^{L^{*}}(f)<\infty$. If $L\left(r^{A}\right)=o\left\{\log T_{h}^{-1} T_{f}\left(r^{A}\right)\right\}$ as $r \rightarrow \infty$ then for any positive number $A$,

$$
\begin{aligned}
\frac{\lambda_{h}^{L^{*}}(f \circ g)}{A \rho_{h}^{L^{*}}(f)} & \leq \liminf _{r \rightarrow \infty} \frac{\log T_{h}^{-1} T_{f \circ g}(r)}{\log T_{h}^{-1} T_{f}\left(r^{A}\right)+L\left(r^{A}\right)} \leq \min \left\{\frac{\lambda_{h}^{L^{*}}(f \circ g)}{A \lambda_{h}^{L^{*}}(f)}, \frac{\rho_{h}^{L^{*}}(f \circ g)}{A \rho_{h}^{L^{*}}(f)}\right\} \\
& \leq \max \left\{\frac{\lambda_{h}^{L^{*}}(f \circ g)}{A \lambda_{h}^{L^{*}}(f)}, \frac{\rho_{h}^{L^{*}}(f \circ g)}{A \rho_{h}^{L^{*}}(f)}\right\} \leq \limsup _{r \rightarrow \infty} \frac{\log T_{h}^{-1} T_{f \circ g}(r)}{\log T_{h}^{-1} T_{f}\left(r^{A}\right)+L\left(r^{A}\right)} \\
& \leq \frac{\rho_{h}^{L^{*}}(f \circ g)}{A \lambda_{h}^{L^{*}}(f)}
\end{aligned}
$$

The proof is omitted.

Combining Theorem a4nd Theorem 6 we may state the following theorem.

Theorem 8. Let $f$ be a meromorphic and $g$ and $h$ be any two entire functions such that $0<\lambda_{h}^{L^{*}}(f \circ g) \leq \rho_{h}^{L^{*}}(f \circ g)<\infty$, and $0<\lambda_{h}^{L^{*}}(g) \leq \rho_{h}^{L^{*}}(g)<\infty$. If $L\left(r^{A}\right)=o\left\{\log M_{h}^{-1} M_{g}\left(r^{A}\right)\right\}$ as $r \rightarrow \infty$ then for any positive number $A$,

$$
\begin{aligned}
\frac{\lambda_{h}^{L^{*}}(f \circ g)}{A \rho_{h}^{L^{*}}(g)} & \leq \liminf _{r \rightarrow \infty} \frac{\log T_{h}^{-1} T_{f \circ g}(r)}{\log T_{h}^{-1} T_{g}\left(r^{A}\right)+L\left(r^{A}\right)} \leq \min \left\{\frac{\lambda_{h}^{L^{*}}(f \circ g)}{A \lambda_{h}^{L^{*}}(g)}, \frac{\rho_{h}^{L^{*}}(f \circ g)}{A \rho_{h}^{L^{*}}(g)}\right\} \\
& \leq \max \left\{\frac{\lambda_{h}^{L^{*}}(f \circ g)}{A \lambda_{h}^{L^{*}}(g)}, \frac{\rho_{h}^{L^{*}}(f \circ g)}{A \rho_{h}^{L^{*}}(g)}\right\} \leq \underset{r \rightarrow \infty}{\limsup } \frac{\log T_{h}^{-1} T_{f \circ g}(r)}{\log T_{h}^{-1} T_{g}\left(r^{A}\right)+L\left(r^{A}\right)} \\
& \leq \frac{\rho_{h}^{L^{*}}(f \circ g)}{A \lambda_{h}^{L^{*}}(g)}
\end{aligned}
$$

Theorem 9. Let $f$ be a meromorphic and $g$ and $h$ be any two entire functions such that $\rho_{h}^{L^{*}}(f)<\infty$. Also let $g$ be entire.If $\lambda_{h}^{L^{*}}(f \circ g)=\infty$ then

$$
\lim _{r \rightarrow \infty} \frac{\log T_{h}^{-1} T_{f \circ g}(r)}{\log T_{h}^{-1} T_{f}(r)}=\infty
$$


Proof. Let us suppose that the conclusion of the theorem do not hold.Then we can find a constant $\beta>0$ such that for a sequence of values of $r$ tending to infinity

$$
\log T_{h}^{-1} T_{f \circ g}(r) \leq \beta \log T_{h}^{-1} T_{f}(r)
$$

Again from the definition of $\rho_{h}^{L^{*}}(f)$ it follows for all sufficiently large values of $r$ that

$$
\log T_{h}^{-1} T_{f}(r) \leq\left(\rho_{h}^{L^{*}}(f)+\varepsilon\right) \log \left(r e^{L(r)}\right) .
$$

Thus from (32) and (33) we have for a sequence of values of $r$ tending to infinity that

$$
\log T_{h}^{-1} T_{f \circ g}(r) \leq \beta\left(\rho_{h}^{L^{*}}(f)+\varepsilon\right) \log \left\{r e^{L(r)}\right\}
$$

That is,

$$
\frac{\log T_{h}^{-1} T_{f \circ g}(r)}{\log \left(r e^{L(r)}\right)} \leq \frac{\beta\left(\rho_{h}^{L^{*}}(f)+\varepsilon\right) \log \left\{r e^{L(r)}\right\}}{\log \left\{r e^{L(r)}\right\}}
$$

So we have,

$$
\liminf _{r \rightarrow \infty} \frac{\log T_{h}^{-1} T_{f \circ g}(r)}{\log r}=\lambda_{h}^{L^{*}}(f \circ g)<\infty .
$$

This is a contradiction.

This proves the theorem.

Remark. Theorem 9 is also valid with "limit superior" instead of "limit" if $\lambda_{h}^{L^{*}}(f \circ g)=\infty$ is replaced by $\rho_{h}^{L^{*}}(f \circ g)=\infty$ and the other conditions remaining the same.

Corollary 1. Under the assumptions of Theorem 9 or Remark 3,

$$
\underset{r \rightarrow \infty}{\limsup _{r \rightarrow}} \frac{T_{h}^{-1} T_{f \circ g}(r)}{T_{h}^{-1} T_{f}(r)}=\infty .
$$

Proof. From Theorem 9 or Remark 3 we obtain for all sufficiently large values of $r$ and for $K>1$ that

$$
\begin{aligned}
\log T_{h}^{-1} T_{f \circ g}(r) & >K \log T_{h}^{-1} T_{f}(r) \\
& >\left\{T_{h}^{-1} T_{f}(r)\right\}^{K},
\end{aligned}
$$

from which the corollary follows.

Theorem 10. Let $f$ be a meromorphic and $g$ and $h$ be any two entire functions such that $\rho_{h}^{L^{*}}(g)<\infty$. Also let $g$ be entire.If $\lambda_{h}^{L^{*}}(f \circ g)=\infty$ then

$$
\lim _{r \rightarrow \infty} \frac{\log T_{h}^{-1} T_{f \circ g}(r)}{\log T_{h}^{-1} T_{g}(r)}=\infty .
$$

We omit the proof of Theorem 10 because it can be carried out in the line of Theorem 9 .

Remark. Theorem 10 is also valid with "limit superior" instead of "limit" if $\lambda_{h}^{L^{*}}(f \circ g)=\infty$ is replaced by $\rho_{h}^{L^{*}}(f \circ g)=\infty$ and the other conditions remaining the same. 
Corollary 2. Under the assumptions of Theorem 10 or Remark 3,

$$
\limsup _{r \rightarrow \infty} \frac{T_{h}^{-1} T_{f \circ g}(r)}{T_{h}^{-1} T_{g}(r)}=\infty
$$

The proof is omitted because it may be carried out in the line of Corollary 1.

\section{Competing interests}

The authors declare that they have no competing interests.

\section{Authors' contributions}

All authors have contributed to all parts of the article. All authors read and approved the final manuscript.

\section{References}

[1] W. Bergweiler : On the growth rate of composite meromorphic functions, Complex Variables, Vol. 14 (1990), pp.187-196.

[2] W. K. Hayman : Meromorphic Functions, The Clarendon Press, Oxford, 1964.

[3] T.V. Lakshminarasimhan : A note on entire functions of bounded index, J. Indian Math. Soc., Vol. 38 (1974), pp. 43-49.

[4] B. K. Lahiri and D. Banerjee : Relative order of entire and meromorphic functions, Proc. Nat. Acad. Sci. India, Vol. 61(A) (2004) No. III, pp.339-354.

[5] S. K. Singh and G. P. Barker : Slowly changing functions and their applications, Indian J. Math., Vol. 19 (1977), No. 1, pp 1-6.

[6] D. Somasundaram and R. Thamizharasi : A note on the entire functions of L-bounded index and L-type, Indian J. Pure Appl.Math., Vol.19 (March 1988), No. 3, pp. 284-293.

[7] G. Valiron : Lectures on the General Theory of Integral Functions, Chelsea Publishing Company, 1949. 